\title{
DEVELOPMENT OF JOINT ATTACHMENT FOR CURVED PIPE PASSAGE OF PERISTALTIC MOTION ROBOT FOR IN-PIPE INSPECTION
}

\author{
TAKUMI YASUI, YUKI MANO, FUMIO ITO, TARO NAKAMURA \\ Faculty of Science and Engineering, Chuo University \\ 1-13-27 Kasuga, Bunkyo-Word, Tokyo 112-8551, Japan \\ *E-mail: t_yasui@bio.mech.chuo-u.ac.jp,y_mano@bio.mech.chuo-u.ac.jp,nakamura@mech.chuo-u.ac.jp
}

\begin{abstract}
In the recent years, aged pipes are increasing, and many road collapse accidents occur. Therefore, it is necessary to inspect the condition of the pipe to efficiently determine whether a replacement or repair is necessary. However, pressure pipes that are capable of coping with complex terrains, the pipelines are complicated and are difficult to inspect with the existing inspection equipment. Therefore, based on the earthworm's peristaltic movement and artificial muscles, the authors developed a peristaltic robot in a previous research for inspecting the inside of a pressure-feeding pipe. However, owing to the unevenness its joint, the robot was unable to maneuver through the corners of a curved pipe. Therefore, in this study, we developed a joint attachment that enables the robot to pass through a curved pipe, and evaluated its effectiveness through traveling experiments by using a curved pipe.
\end{abstract}

\section{Introduction}

In recent years, sewer maintenance has been promoted; as of 2013, pipeline extensions have increased to approximately $460,000 \mathrm{~km}$. Meanwhile, a rapid increase in the number of aged pipes that have been installed for 50 years is expected. Damages to these old pipes can cause serious accidents such as road collapse, and urgent measures are required [1]. However, it is difficult to repair or replace all old pipes immediately owing to budget restrictions. Therefore, it is effective to inspect the condition in the piping to efficiently find the piping and repair point to be replaced preferentially.

The primary method for surveying sewage pipes is using a push-in type endoscope that pushes the endoscope into the pipe manually. This method affords an easy in-pipe inspection. However, it is difficult to inspect pipes with complicated arrangements such as pressure pipes and sewer pipes. This is because the method of pushing the endoscope causes friction to occur between the endoscope and pipe when inspecting a pipe in which a plurality of curves and the like exist. Consequently, the pushing force at the hand is not transmitted to the head, and long-distance inspections of $30 \mathrm{~m}$ or more are difficult to perform.

Hence, research on in-pipe inspection robots is conducted actively as an inspection method to replace endoscopes. Many involve robots with wheels [2-7]. In addition, robots that move using vibration are being developed [8]. Wheeled robots are required to pull power and camera cables over long distances. However, when the wheeled robots output this pulling force, they become larger. Therefore, it is considered difficult for wheeled robots to use a long-distance smalldiameter pipe inspection with a diameter of $150 \mathrm{~mm}$ or less. Because a ciliary robot is covered with thin hair throughout its body, friction with the pipe wall is reduced. Because they cannot grip the pipe sufficiently, traction is reduced. Therefore, a ciliary vibrating robot is considered not suitable for long-distance pipe movements because it is difficult to apply these robots to the inspection of pressure pipes.

Hence, the authors focused on an earthworm's peristaltic movement that can be applied to narrow places. We developed a robot, PEW-RO, that simulates the peristaltic movement of earthworms; it traveled through a pipe with an inner diameter of $108 \mathrm{~mm}$, and passed through a curved pipe successfully [9]. The joint of PEW-RO V, the latest version of PEW-RO, mimics a 
universal mechanism [10]. The mechanism is a structure with a high degree of freedom of joints for passage through a curved pipe. However, universal joint gap can occur on the steps inside the curved pipe. Therefore, in this study, a joint attachment is developed to eliminate the unevenness of the universal mechanism, and the influence of this joint attachment on the curved pipe passage performance is evaluated from a curved pipe traveling test.

\section{Present state of pressure pipe inspection}

A pressure pipe can transport liquid by the pressure of a pump, and its layout is not restricted to the topography. Therefore, the pipeline is arranged in a complicated state. Because the longterm use of the pressure pipe causes internal defects, inspection is necessary. Conventional methods that use an endoscope cannot easily inspect if multiple curves exist. Therefore, inspection robots have been developed. A pressure-pipe inspection robot is required to inspect a long-distance pipe with multiple curves. However, owing to problems such as the lack of traction due to miniaturization, the developed robot is considered difficult to use. Therefore, the authors propose a robot that simulates an earthworm's peristaltic movement. In this case, the pressure pipe to be targeted is $100 \mathrm{~m}$ in length and three curves, and an inspection within $2 \mathrm{~h}$ (7200 s) is necessary to account for the halt in sewage flow in the pressure pipe at the time of inspection. Therefore, we aim to inspect the entire pipeline at an average speed of $27.8 \mathrm{~mm} / \mathrm{s}$ or more.

\section{Peristaltic motion robot PEW-RO V}

\subsection{Outline of peristaltic movement robot PEW-RO V}

We developed a peristaltic robot PEW-RO $\mathrm{V}$ that mimics the peristaltic movement of earthworms. The appearance of PEW-RO V is shown in Figure 1. PEW-RO V consists of a unit, joint, head, and tail-following solenoid valve, with a total length of $1700 \mathrm{~mm}$ and weight of $6500 \mathrm{~g}$.

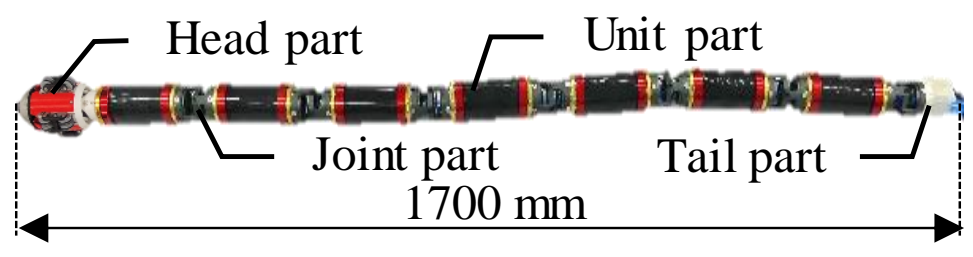

Figure 1. Peristaltic crawling robot PEW-RO V

\subsection{Unit part}

The unit of this robot contracts and inflates through artificial muscles(Figures 2 and 3). The peristaltic movement is reproduced by propagating this contraction and expansion in the order from the front to the rear, and it travels through the pipe. A pressing ring is used to fix the artificial muscle. Furthermore, the artificial muscle is fixed by fastening the pressing ring with a nut (Figure 4). Using this method, it is possible to prevent the falling out of the artificial muscle by intensifying the expansion suppression of the artificial muscle. 


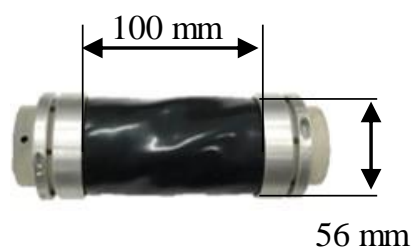

(a)Normal state

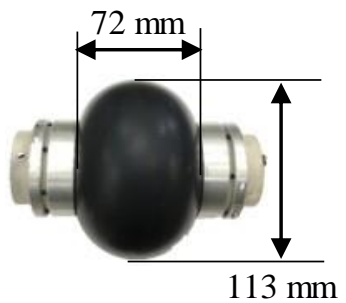

(a)Contraction state

Figure 2. Unit part of PEW-RO V

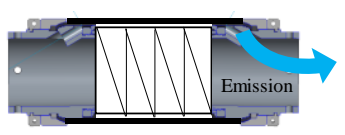

(a)Normal state

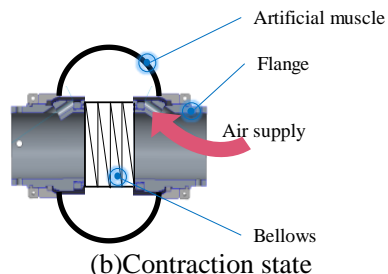

(b)Contraction state

Figure 3. Cross-sectional view of unit part of PEW-RO V

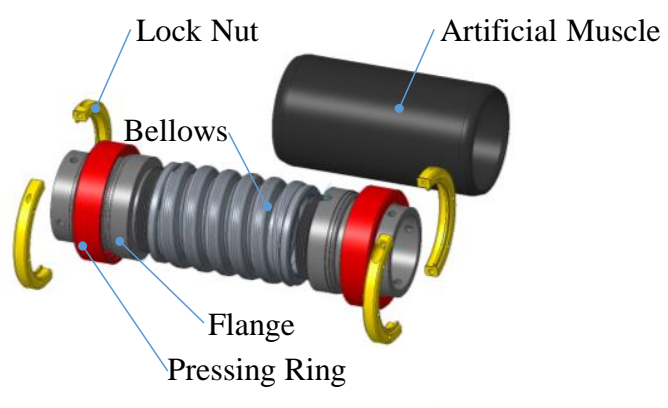

Figure 4. Structure of unit part

\subsection{Joint part}

This joint is a universal joint with a hollow inside (Figure 5). And has a high degree of freedom. In addition, by increasing the rigidity of the joint using a pressure spring contained inside, the buckling of the entire robot is eliminated and the curved pipe passage performance is improved. However, owing to the unevenness in this joint, it may be caught on a step in the pipe when passing through a curved pipe. Therefore, we propose an improvement to this joint.

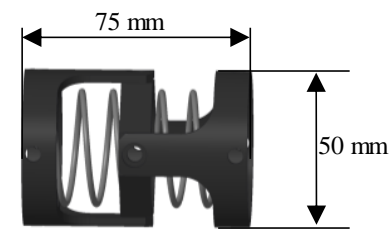

(a)Side view of joint part

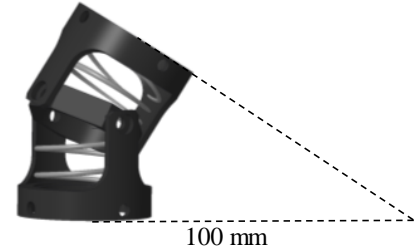

(b)Curving state of joint part

Figure 5. Joint part of PEW-RO V 


\subsection{Tail-following solenoid valve and drive system}

Figure 6. shows a schematic diagram of the drive system of PEW-ROV. Air supplied from an air supply source is supplied to each unit of PEW-ROV through a solenoid valve controlled by a microcomputer. The solenoid valve opens and closes the air flow path by the signal sent from the microcomputer. Thus, air can be supplied and exhausted independently to each unit. This solenoid valve is collected in the tail part and placed on the cable. Having a sufficient effective cross-sectional area of the solenoid valve leads to the speed improvement of PEW-RO V [10]. Therefore, for PEW-ROV, a solenoid valve (B3015-3-BB-LB-C204) manufactured by Danaher, which can produce the maximum effective cross-sectional area according to the inner diameter of the pipe, is used. Furthermore, two direct acting three-port solenoid valves are used in one unit part. This enables the air supply and exhaust time to be reduced [11].

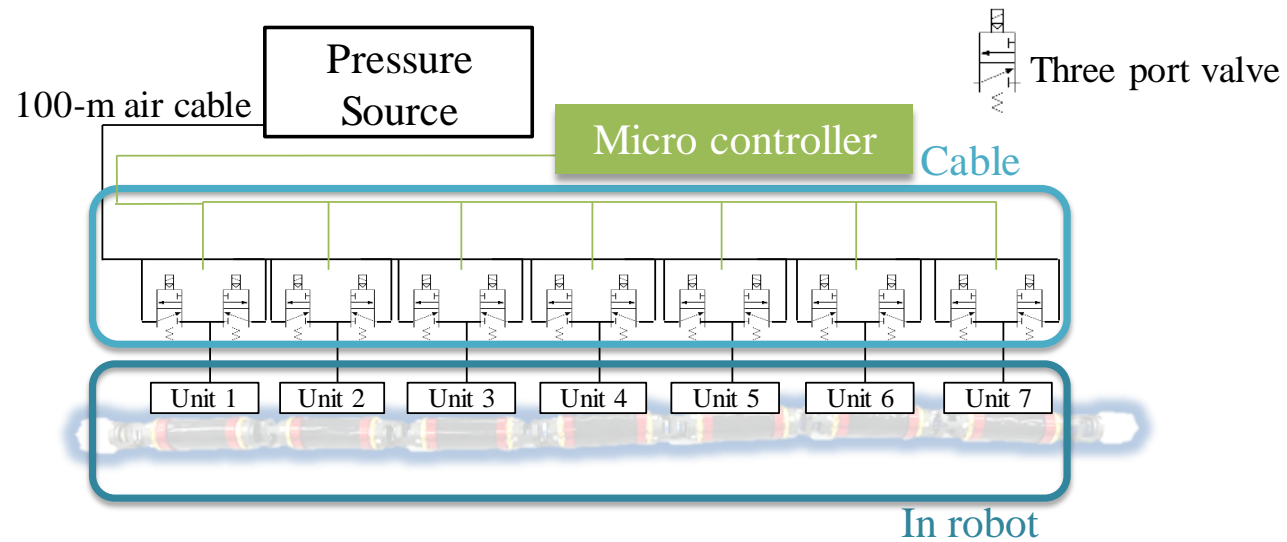

Figure 6. Schematics of PEW-RO system

\section{Proposal of joint attachment}

This section describes the joint attachment for the passage through a curved pipe. The joint described in Section 3.3 has the freedom to curve the curved pipe; however, we confirmed that the joint gap was caught at the corner of the curved pipe. Therefore, a mechanism that prevents the joint from being caught is necessary. As the mechanism must cope with the curving of the joint, a soft material could be effective. Therefore, we used urethane foam, whose elasticity can prevent the joint from biting into the corner without inhibiting the curving of the joint. By winding it around the joint, hooking could be prevented when it collided with the corner of the curved pipe. However, friction could occur between the elastic body and pipe wall. Therefore, a nylon brush was used as a low-friction material around the circumference of the elastic body to prevent friction with the pipe wall (Figure 7). This brush does not interfere with the curved pipe angle because it has a collapsible structure.

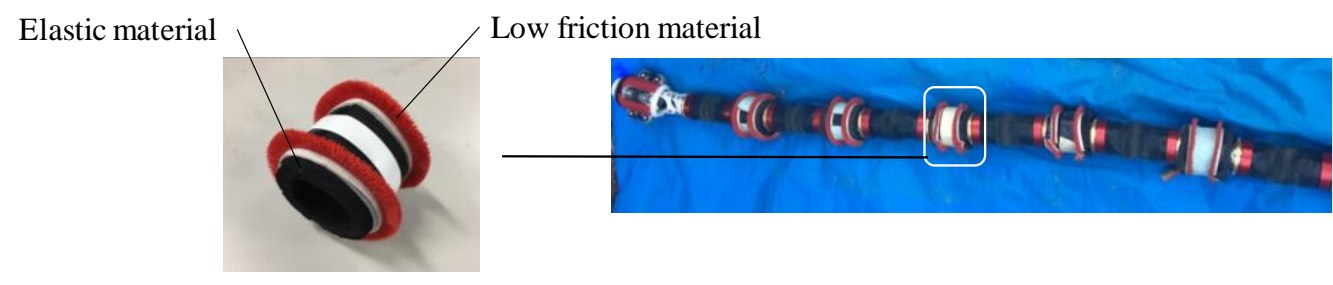

Figure 7. Joint attachment 


\section{Curved pipe passage performance evaluation experiment of joint attachment}

To confirm whether the proposed joint attachment is effective in passing through a curved pipe, a traveling experiment of a $90^{\circ}$ curved pipe of PEW-ROV was performed. We conducted experiments on two standardized $90^{\circ}$ pipes (Figure 8) with the largest curving angle, in the pressure pipe. For the experiment, we used an acrylic pipe that simulated a 100A pipe (108 mm in diameter), which is generally used in a pressure pipe.

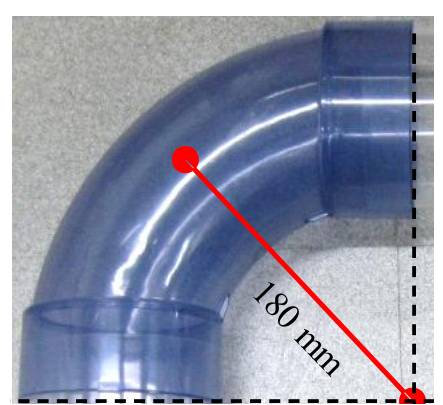

(a)Large bent pipe

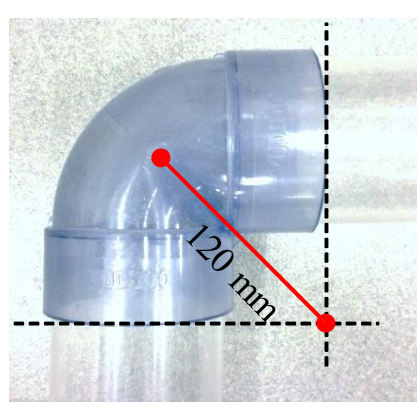

(b) $90^{\circ}$ elbow pipe

Figure 8 . Types of $90^{\circ}$ bent pipe

\subsection{Setting the experimental environment and time required to pass through the curved pipe}

Figure 9. shows a schematic of the experimental environment. Air pressure is supplied from the air compressor, and the direct acting solenoid valve opens and closes in response to a command from the microcomputer. Measurement starts when the head of the robot curves into the pipe and ends when the tail of the robot passes through the curve. The applied base pressure was 0.36 $\mathrm{MPa}$. The tow cable was $100 \mathrm{~m}$; however, $20 \mathrm{~m}$ of it was used for simplicity. All the pipes used were straight horizontal pipes that were sufficient long for the robot to move. The number of trials was five for both the large curve and elbow.

We subsequently set the curve passage target time. The total length of the target pressure pipe is $\mathrm{L}$ [mm], the number of curving pipe $\mathrm{s}$ is $\mathrm{N}$, the target inspection time is $\mathrm{S}$ [s], the total length of the robot is $1[\mathrm{~mm}]$, and the average velocity of the robot is $\mathrm{V}[\mathrm{mm} / \mathrm{s}]$, The curved pipe passage target time $\mathrm{T}$ [s] can be calculated using Eq. (1). In this case, the pressure pipe to be targeted is reciprocation $\mathrm{L}=200000 \mathrm{~mm}$, curving point in reciprocation $\mathrm{N}=6$ and inspection target time $\mathrm{S}=7200 \mathrm{~s}$. Furthermore, $\mathrm{T}=270 \mathrm{~s}$ when the robot's total length $1=1700 \mathrm{~mm}$ and the robot's horizontal straight pipe velocity $\mathrm{V}=34 \mathrm{~mm} / \mathrm{s}$.

$$
T=\frac{S V-(L-N l)}{N V}
$$




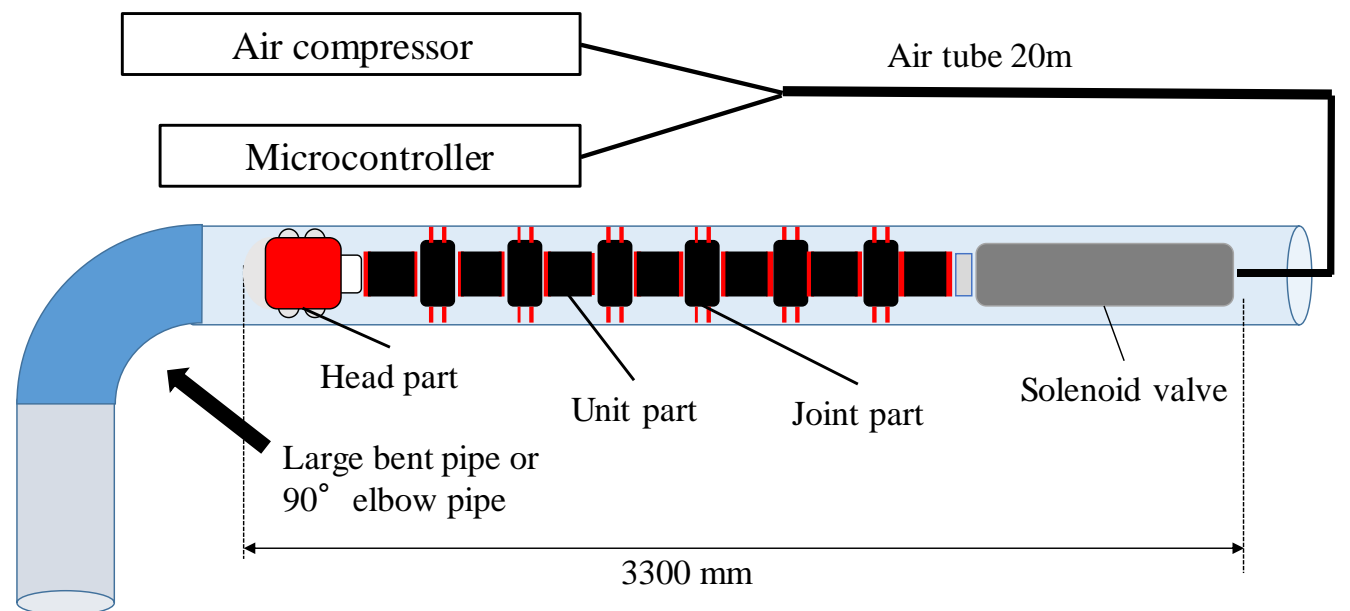

Figure 9. Schematic diagram of the experiment

\subsection{Experimental result and consideration}

Figures 10 and 11. show how the large bent pipe and elbow pipe ran with the joint attachment, respectively. In addition, Figure 12. shows the relationship between the number of trials and transit time of the large curved pipe and elbow pipe. The large bent pipe required a longer time than the other trials in the second trial; however, we confirmed that it was less than $270 \mathrm{~s}$, which was the target time for passing through the curved pipe, in all trials. In addition, there was no significant difference in transit time for the elbow pipe, and it fell below the curve passage target time of $270 \mathrm{~s}$ in all trials. The video captured in the experiment confirmed that no catching occurred. Therefore, we confirmed that the elastic body prevented the joints from being caught. In addition, the passage performance was practical because the passage time was less than that required in all trials.

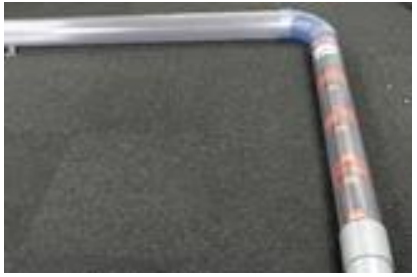

(a) $0[\mathrm{~s}]$ (start)

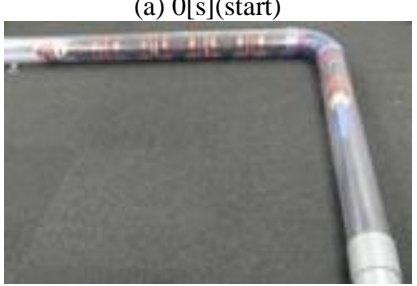

(c) $60[\mathrm{~s}]$

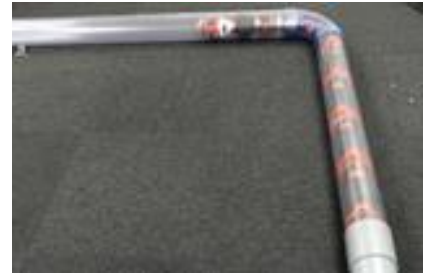

(b) $30[\mathrm{~s}]$

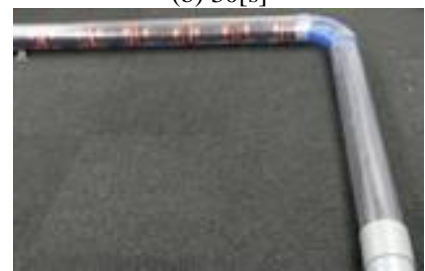

(d) $87[$ s](finish)

Figure 10. Driving test in large curve pipe 


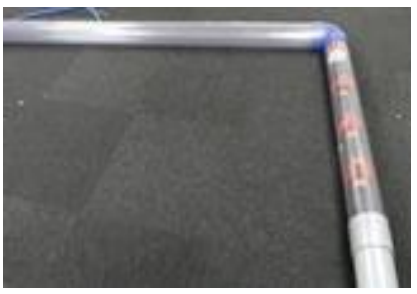

(a) $0[\mathrm{~s}]($ start)

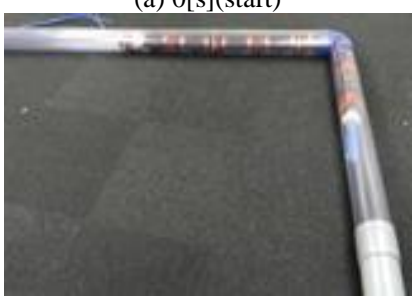

(c) $100[\mathrm{~s}]$

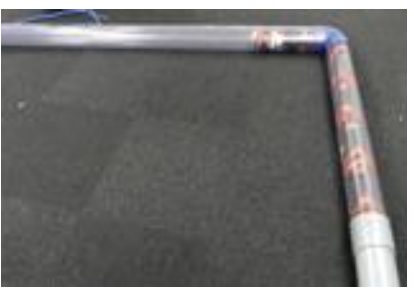

(b) $50[\mathrm{~s}]$

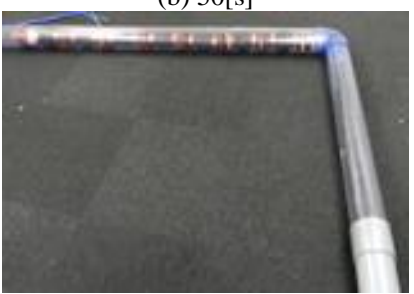

(d) $162[\mathrm{~s}]($ finish)

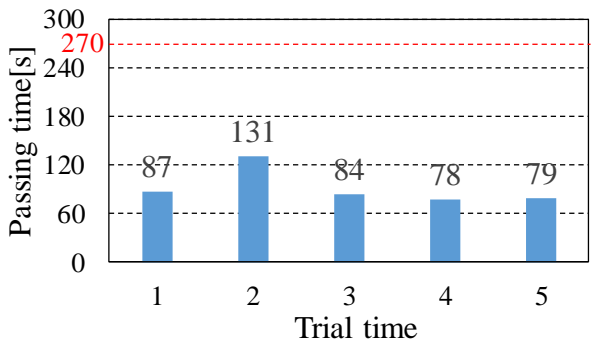

(a) Passing time of large bent pipe

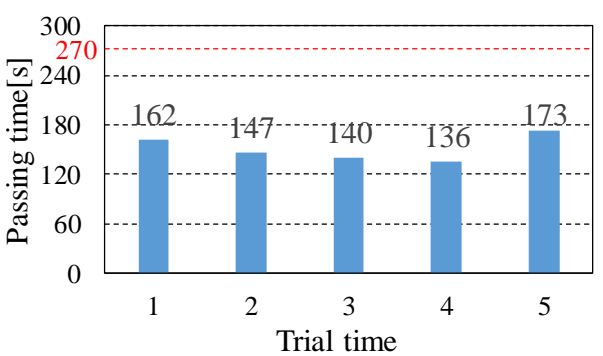

(b) Passing time of elbow pipe

Figure 12. Passing time of $90^{\circ}$ bent pipe

\section{Conclusion}

In this study, we developed a joint attachment for a robot that combined an elastic polyurethane foam and a low-friction nylon brush. Furthermore, the PEW-ROV was attached to the joint attachment, and a passage experiment was performed with a curved pipe. The experiments, demonstrated success at a $90^{\circ}$ curve, and the usefulness of the developed joint attachment was confirmed. In this study, a low-friction nylon brush was attached on the circumference of the robot body. In the future, we will examine how the nylon brush arrangement affects the passage through the curved pipe.

\section{References}

1. H. Uchida and K. Ishii, Proc. 2009 JSME Conf. Robotics and Mechanics, 09-4 (2009).

2. J.Y. Nagase and F. Fukunaga, IECON 2016 - 42nd Annual Conference of the IEEE Industrial Electronics Society.

3. T. Miyagawa, K. Suzumori, M. Kimura and Y. Hasegawa, J. of RSJ, 17,389 395,(1999).

4. J. J. Park, J. W. Moon, H. Kim, S. C. Jang, D. G. Kim, K. Ahn, S. M. Ryew, H. Moon and H. Ryeol, 5. Z. Yunwei and G. Yan, Mechanism and Machine Theory 42.12 1618-1631, (2007).

6. M. A. A. Wahed and M. R. Arshad, 2017 IEEE 2nd International Conference on Automatic Control and Intelligent Systems (I2CACIS).

7. P. Debenest, M. Guarnieri and S. Hirose, Proceedings of the 2014 3rd International Conference on Applied Robotics for the Power Industry. 
8. K. Hatazaki, M. Konyo, K. Isaki, S. Tadokoro and F. Takemura, Proceedings of the 2007 IEEE/RSJ International Conference on Intelligent Robots and Systems, (2007).

9. T. Tomita, T. Tanaka and T. Nakamura, IEEE/RSJ International Conference on Intelligent Robots and Systems, (2015).

10. Y. Mano, R. Ishikawa, Y. Yamada and T. Nakamura, IEEE/RSJ International Conference on Intelligent Robots and Systems (IROS), (2018).

11. Y. Mano, R. Ishikawa, Y. Yamada and T. Nakamura, IEEE/ASME International Conference on Advanced Intelligent Mechatronics (AIM), (2018). 